\title{
ONE YEAR -RANDOMIZED CLINICAL TRIAL TO EVALUATE PACKABLE BULK FILL AND NANO RESIN COMPOSITE IN CHILDREN AND ADOLESCENTS
}

\author{
Shaimaa M. Mahfouz* and Asmaa A.Emam*
}

\begin{abstract}
Purpose: To evaluate the one- year clinical performance of the packable bulk fill and nano resin composite in class I restorations in children and a adolescents.

Materials \& Methods: (33) pairs of first permanent molars had class I restorations were placed in (33) children were selected from Outpatient Clinic, Pediatric Dentistry Department, Faculty of Dentistry, Suez Canal University, each patient received two class I restorations that were similar as possible. In all cavities, single bond adhesive (Xeno V+) was applied. One cavity of each pair was randomly assigned to be restore with packable bulk fill resin composite (Quixfil) in increment up to $4 \mathrm{~mm}$. Another cavity was restored with Nano hybrid resin composite (Esthet-X flow) placed in $2 \mathrm{~mm}$ increments. All restorations were evaluated at one week baseline and then one month, 3 months, 6 months , 9 months and 12 months . using slightly modified USPHS .
\end{abstract}

Results: At all periods of evaluation, no statistically significant differences were detected between packable bulk fill composite resin and Nano hybrid composite resin in marginal adaptation, surface roughness and caries detection, whereas statistically significant differences between both materials in anatomical form and marginal discoloration parameters in favor with packable bulk fill. Sensitivity was not reported during all evaluation periods Performance at one week base line, 1 month, 3 months, 6 months, 9 months and 12 months for all criteria.

Conclusions: The packable bulk- fill composite resin showed highly clinical performance when compared with Nano hybrid composite resin restorations at one- year follow up. Further studies necessary for long term clinical evaluation of these materials.

KEY WORDS: Bulk fill- Nano composite

\section{INTRODUCTION}

Increasing attention on the aesthetic abilities of dental restorations and improvements in resin technologies that are accessible, composite resin restorative materials are being used for posterior teeth as an alternative of amalgam restoration (1). Additionally, as a result of advances in adhesive properties thatcausing reductionincavity preparation

\footnotetext{
* Lecturer of Pediatric and Preventive Dentistry and Dental Public Health ,Faculty of Dentistry, Suez Canal University.
} 
size, many of the composite resin restorative materials used in current restoration have the capacity to strengthen the remaining tooth structure far more than conventional amalgam restoration(2) .Composite resin restoration can be placed into cavity by different approaches, many studies used layering technique in which the material can be placed in increments of 2-mm or less (3) . This way results in adequate light infiltration and improved polymerization of composite resin materials (4). Also, this will lead to decrease polymerization shrinkage as well as cuspal deflection (5). Bulkfill resin composites introduced into cavities in one increment that up to $4-\mathrm{mm}$, this thickness offers several advantages of saving time and simplifying technique of placement the restoration (6). Many in vitro studies revealed that application of denser increment could be equally effective as conventional increments technique (4). There is no clinical study that reported the efficiency of packable bulk- fill composite resin with maximum $4 \mathrm{~mm}$ depth in class I cavity in children. Therefore, the aim of the present study was to clinical evaluate the packable bulkfill composite resin and nano hybrid composite resin restoration which was used as positive control group . The null hypothesis was that packable bulkfill composite had the same clinical presentation as nano hybrid composite resin.

\section{MATERIALS AND METHODS}

A total (33) Normal, apparently healthy and cooperative children adolescences aged from (914) years and they were selected from Outpatient Clinic, Pediatric Dentistry Department, Faculty of Dentistry, Suez Canal University. The patient general information, including name, gender and age were recorded. Medical and dental histories were taken. A preoperative bitewing radiographs was taken to exclude proximal caries. Patients had at least one pairs of similar class I permanent first molar. The reasons for restoring permanent molars were primary or secondary caries lesions.

\section{Inclusion criteria (6)}

- Vital tooth

- Tooth did not have any signs or symptoms of pulpal and periapical disease.

- Tooth to be restored should be at occlusion.

- Absence of oral habit.

- Good oral hygiene.

- Good condition of periodontal tissues (6).

\section{Exclusion criteria (6)}

- Non vital tooth .

- Tooth has any signs or symptoms of pulpal and periapical disease.

- Tooth to be restored out of occlusion.

- Presence of oral habit.

- Bad oral hygiene.

- Bad condition of periodontal tissues.

- Uncooperative child

- Parent refuse informed consent

- Allergy to the materials used in this trial (6).

Treatment was explained to parents and written informed consent was obtained before the study (As in Appendix). Adequate diagnosis and treatment planning were formed. Each patient had similarity cavity size and depth as possible. Each patient had cavities more than $2 \mathrm{~mm}$. depth. Molars had class $\mathrm{V}$ according to ICDAS* . Cavity preparation was performed by diamond bur ${ }^{* *}$ using high speed with water coolant. Local anesthesia may be needed in patients with discomfort or pain during operative

*International caries detection assessment system

**3M ESPE, Seefeld, Germany. 
procedure. Adequate isolation with rubber dam and high suction were used. Only caries or defective restoration removed without any beveling of walls and without any extensions. Adequate dryness of cavity performed by ship syringe then using single bond (Xeno V) and curing for $20 \mathrm{~s}$. flowable composite (Esthet-X flow) was applied as a cavity liner then either packable bulk fill (Quixfil)or Nano hybrid composite (Ceram X mono) were applied according to treatment modalities of each group (table 1). Bulk fill composite applied as one increment then curing for $10 \mathrm{~s}$ while Nano composite were applied in increments, $2 \mathrm{~mm}$ maximum thickness for each layer then curing for $20 \mathrm{~s}$ then applying other layer then curing for $20 \mathrm{~s}$. Restorations were finished with fine green stones polished with cups and points after $24 \mathrm{~h}$. proper occlusal morphology was created by using Articulating paper*. LEDcuring unit ${ }^{* * *}$ was used for light-curing processes that is performed in a continuous mode, releasing a light intensity of $1200 \mathrm{~mW} / \mathrm{cm}^{2}$. Molar had been divided into two groups according to treatment modalities into group I Permanent molar had been filled with packable bulk fill posterior resin Composite and TABLE (1) Showing all materials used in the study
Group II Permanent molar had been filled with nano hybrid composite resin (fig1) (fig2).

Operative procedures were accomplished by one experienced dentist. Everyone had received similar sized and positioned restoration as possible. Before starting operative process, the two cavities in each individual were randomly distributed to be filled with either the experimental restoration or positive control restoration. The participants were not aware in which cavity, the experimental and control restoration were placed.

Different Materials were used in this study summarized in Table (1)

\section{Clinical evaluation:}

All restorations were clinically assessed by using "Modified US Public Health Service" (USPHS) (2). Ryge criteria for retention, marginal adaptation, anatomic form, surface texture and secondary caries [Table 2]. One examiner who was not involved in the placement of the restorations evaluated the restorations after 1 week (baseline), 1 month, 3 months, 6 months, 9 and 12 months.

\begin{tabular}{|c|c|c|}
\hline Name of product & Name of company & Material composition \\
\hline Quixfil & Dentsply Caulk, Germany & $\begin{array}{l}\text { Bulk fill Posterior resin composite } \\
\text { UDMA, TEGDMA, di- and trimethacrylate resins, carboxylic acid } \\
\text { modified dimethacrylate resin BHT, UV stabilizer }\end{array}$ \\
\hline Esthet-X flow & Dentsply Caulk, Germany & $\begin{array}{l}\text { Liquid flowable microhybrid Urethane modified BisGama and diluents } \\
\text { Camphoroquinone photoinitiator;stabilizer;pigments. } \\
\text { Filler:-Bariumfluoroaqluminum borosilicate glass with nanofillersilica }\end{array}$ \\
\hline Xeno V+ & Dentsply Caulk, Germany & One component one step self - etching adhesives \\
\hline Ceram $\mathrm{X}$ mono + & Dentsply Caulk, Germany & $\begin{array}{l}\text { Filler:Barium- aluminum borosilicate glass }(1.1 .-1.5 \mu \mathrm{m}) \text {, methacrylate } \\
\text { functionalized silicone dioxide nano filler }(10 \mathrm{~nm}) \text {. filler content w: } 76 \% \\
\text { v: } 57 \% \text { filler content. Matrix Methacrylate modified polysiloxane } \\
\text {,dimethacrylate resin ethyl-4-(dimethylamino) benzoate florescent } \\
\text { pigment, UV stabilizer, stabilizer, camphorquinone, titanium oxide } \\
\text { pigments, alumuinum silicate pigments. }\end{array}$ \\
\hline
\end{tabular}

\footnotetext{
* Bausch, Nashua, NH, USA

** Elipar S10; 3M ESPE, Seefeld, Germany
} 


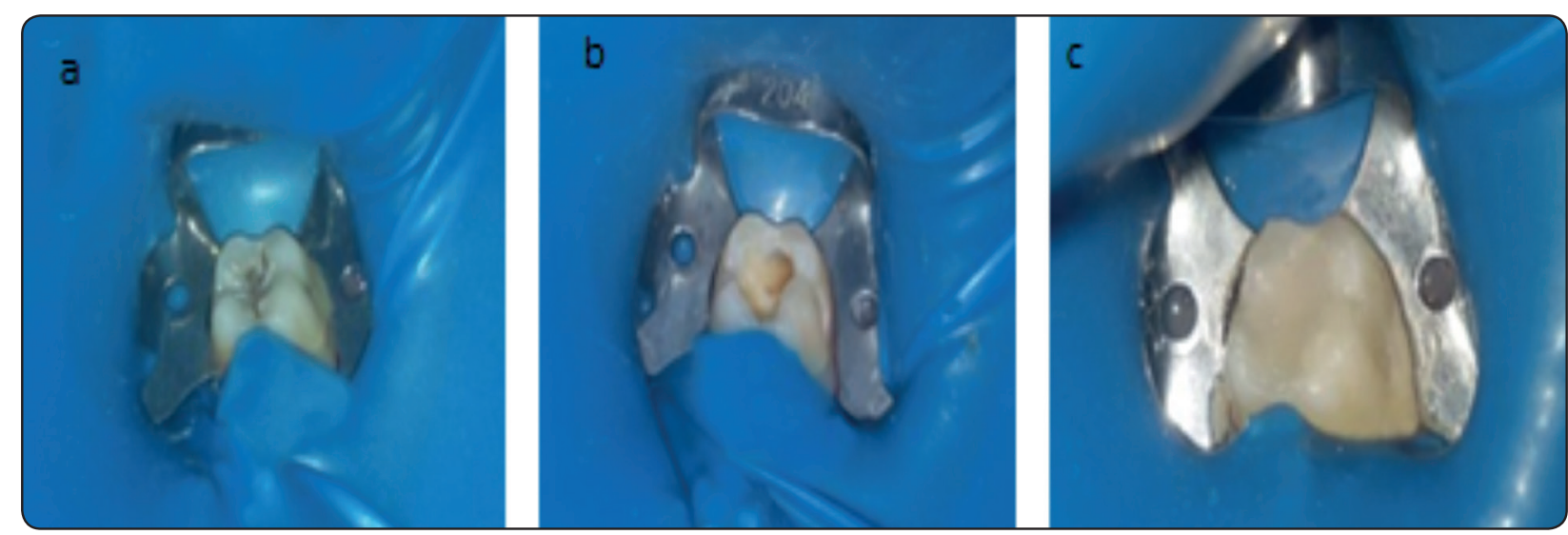

Fig. (1) a) preoperative carious permanent molar. b) Cavity preparation. c) Bulk fill composite resin restoration.
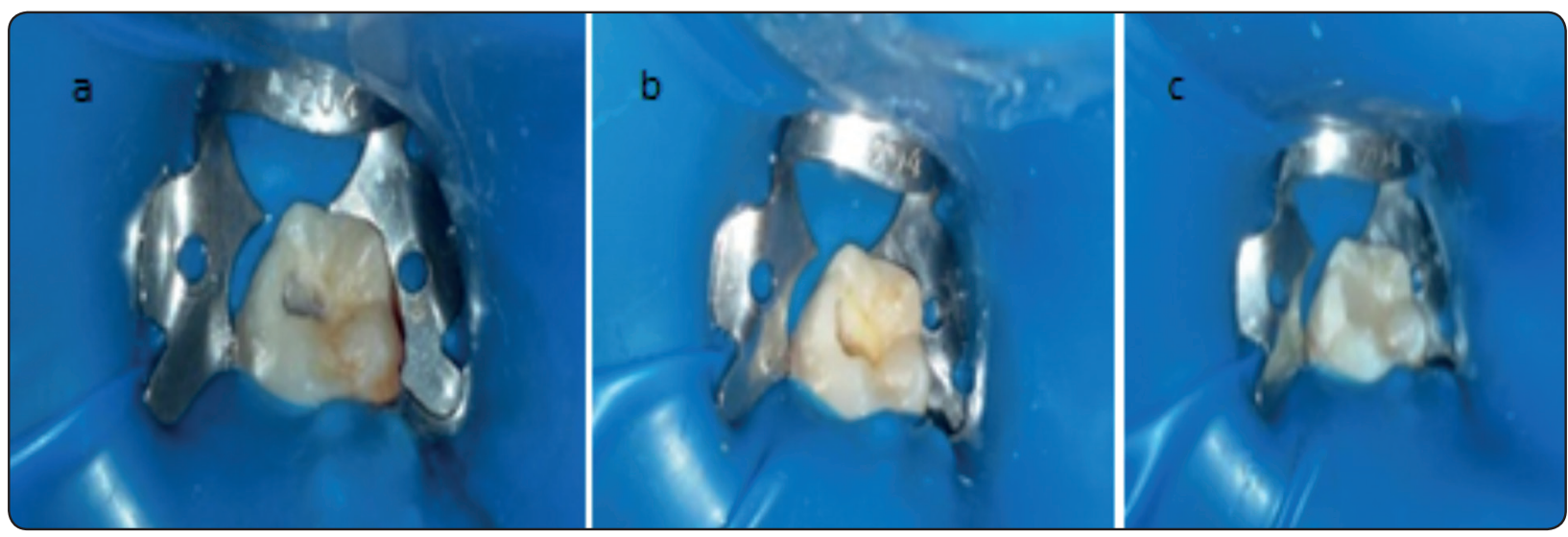

Fig. (2) a) preoperative carious permanent molar. b) Cavity preparation. c) Nano composite resin restoration.

\section{RESULTS}

From November 2016 to Mai 2018, in the present study 66 restorations were performed in 33 patients. Patient's ages ranged from 9 to 14 years. Three patients (6 restorations) were lost during one month recall visit. The results obtained after all evaluation periods are shown in table (3). Patients were re-evaluated at 1 week baseline, 1 month, 3 , 6, 9 and 12 months. No post-operative symptoms were reported at baseline or at the other recall. The scores at 1 week baseline and 1 month, 3, 6, 9 and 12 months for all evaluated restorations are given as relative frequencies (\%) in table (3) . The modified USPHS scores of bulk fill and Nano hybrid composite are given in table (2).

\section{Anatomical form:}

The results showed that there were no differences in scores and frequencies at baseline between both groups (table 3) At one week and one month recall 66 restorations, bulk fill and nano hybrid composite score $100 \%$ (score 0) was recorded. Also at 3 months recall 30 restorations, bulk fill and nano hybrid composite score 70\% (score 0) and score $30 \%$ (score 1) were recorded. On other hand at 6 months recall 30 restorations, bulk fill and nano hybrid composite score $50 \%$ (score 0 ) and score $50 \%$ (score 1) were recorded. In addition at 9 months recall 30 restorations, bulk fill and nano hybrid composite score $40 \%$ (score 0 ) and score $60 \%$ (score 1) were recorded. Also at 12 months recall 
TABLE (2) Showing modified (USPHS) for evaluation of restorations

\begin{tabular}{|c|c|c|}
\hline Category & Scores & Criteria \\
\hline Anatomic form & $\begin{array}{l}0 \\
1 \\
2 \\
3\end{array}$ & $\begin{array}{l}\text { - The restoration is contiguous with tooth anatomy } \\
\text { - Slight under or over contoured; marginal ridge slightly undercontoured; occlusal } \\
\text { hight reduced locally. } \\
\text {-Restoration is undercontoured, dentin or base exposed ;occlusal height reduced; } \\
\text { occlusion affected. } \\
\text { - Restoration is missing partially or totally ; fractured tooth structure;shows } \\
\text { traumatic occlusion ; restoration causes pain in tooth or adjacent tissue. }\end{array}$ \\
\hline Marginal discoloration $\mathrm{m}$ & $\begin{array}{l}1 \\
2 \\
3 \\
4\end{array}$ & $\begin{array}{l}\text {-Absence of marginal discoloration } \\
\text {-Slight staining can be polished away. } \\
\text {-obvious staining can not polished away. } \\
\text { - Gross staining }\end{array}$ \\
\hline Marginal adaptation & $\begin{array}{l}0 \\
1 \\
2 \\
3 \\
4\end{array}$ & $\begin{array}{l}\text { - Restoration is contiguous with existing anatomic form, explorer does not catch. } \\
\text {-Explorer catches. No cervice is visible into which explorer will penetrate } \\
\text { - crevice at margin, enamel exposed. } \\
\text {-Obvious crevice at margin, dentin or base exposed. } \\
\text {-Restoration mobile, fractured or missing }\end{array}$ \\
\hline Surface roughness & $\begin{array}{l}0 \\
1 \\
2 \\
3\end{array}$ & $\begin{array}{l}\text {-Smooth surface } \\
\text {-Slight rough or pitted. } \\
\text { - rough can not be finished. } \\
\text { - Surface deeply pitted }\end{array}$ \\
\hline Secondary caries & $\begin{array}{l}0 \\
1\end{array}$ & $\begin{array}{l}\text { No caries } \\
\text { Caries }\end{array}$ \\
\hline
\end{tabular}

30 restorations, bulk fill and nano hybrid composite score $30 \%$ (score 0 ) and score $70 \%$ (score 1) were recorded. Statistical analysis showed statistically significance differences between both materials in favor with bulk fill (Quixfil) p- value $=0.001$

\section{Marginal adaptation:}

At one week and one month recall 66 restorations both bulk fill and nano hybrid composite score $100 \%$ (score 0) was recorded but Also at 3 months recall 30 restorations, bulk fill and nano hybrid composite score $70 \%$ (score 0 ) and score $30 \%$ (score 1) were recorded. On other hand at 6 months recall 30 restorations, bulk fill and nano hybrid composite score $70 \%$ (score 0 ) and score $30 \%$ (score 1) were recorded. In addition at 9 months re- call 30 restorations, bulk fill and nano hybrid composite score $60 \%$ (score 0 ) and score $40 \%$ (score 1) were recorded. Also at 12 months recall 30 restorations both bulk fill and nano hybrid composite score 50\% (score 0) , 40\% (score 1) and 10\% (score 4) were recorded. Statistical analysis showed no statistically significance differences between both materials $p$ - value $=0.106$

\section{Marginal discoloration:}

At one week and one month recall 66 restorations both bulk fill and nano hybrid composite score $100 \%$ (score 0) was recorded. In addition at 3 months recall 30 restorations, bulk fill score 90\% (score 0), score 10\%(score 1) and 30 restorations nano hybrid composite score $80 \%$ (score 0 ) and score $20 \%$ (score 1) were recorded. Whereas in 
6 months recall 30 restorations bulk fill score $70 \%$ (score 0 ) , score 30\%(score 1) also, 30 restorations nano hybrid composite score $70 \%$ (score 0 ) and score $30 \%$ (score 1) were recorded. At 9 months recall 30 restorations bulk fill score $60 \%$ (score 0 ), $30 \%$ (score 1), $10 \%$ (score 2) and 30 restorations nano hybrid composite score $60 \%$ (score 0 ), score $20 \%$ (score 1) and score $20 \%$ (score 2) were recorded. Also at 12 months recall 30 restorations bulk fill score 60\% (score 0), score 20\% (score 1), $20 \%$ (score 2) and 30 restorations nano hybrid composite score $40 \%$ (score 0), score $40 \%$ (score 1) and score $20 \%$ (score 2) were recorded. Statistical analysis showed statistically significance differences between both materials in favor with bulk fill (Quixfil) p- value $=0.008$.

\section{Surface roughness:}

At one week and one month recall 66 restorations both bulk fill and nano hybrid composite score $100 \%$ (score 0) was recorded. At 3 months recall 30 restorations, bulk fill score $90 \%$ (score 0 ), score 10\%(score 1) and 30 restorations nano hybrid composite score $80 \%$ (score 0) and score 20\% (score 1) were recorded. Whereas at 6 months recall 30 restorations, bulk fill score $80 \%$ (score 0), score $20 \%$ (score 1) and 30 restorations nano hybrid composite score $70 \%$ (score 0 ) and score 30\% (score 1) were recorded. At 9 months recall 30 restorations, bulk fill score $70 \%$ (score 0 ), score $30 \%$ (score 1) and 30 restorations nano hybrid composite score $60 \%$ (score 0 ), score $30 \%$ (score 1 ) and $10 \%$ (score 2 ) were recorded. In addition at 12 months recall 30 restorations, bulk fill score $60 \%$ (score 0 ), score $30 \%$ (score 1), score $10 \%$ (score 2) and 30 restorations nano hybrid composite score $60 \%$ (score 0 ) and score $20 \%$ (score 1) and score 20\% (score 2) were recorded. Statistical analysis showed no statistically significance differences between both materials $\mathrm{p}$ - value $=0.102$.

\section{Caries}

At 1 week , 1 ,3, 6 month recall 66 restorations both bulk fill and nano hybrid composite score
$100 \%$ (score 0) was recorded. While at 9 months recall 30 restorations, bulk fill score 100\% (score 0 ) and 30 restorations nano hybrid composite score $90 \%$ (score 0 ) and score $10 \%$ (score 1) were recorded. In addition at 12 months recall 60 restorations bulk fill and nano hybrid composite score $90 \%$ (score 0 ), score 10\%(score 1) were recorded. Statistical analysis showed no statistically significance differences between both materials $\mathrm{p}$ - value $=0.46$

TABLE (3) showing different scores for evaluation of (Quixfil) and (Ceram X mono+) at all evaluation periods $(n=33$ and 33 ) given as relative frequencies $(\%)$

\begin{tabular}{|c|c|c|c|c|c|c|}
\hline \multirow{2}{*}{ 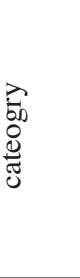 } & \multirow[t]{2}{*}{$\begin{array}{l}\text { Evalu- } \\
\text { ation } \\
\text { period }\end{array}$} & \multirow[t]{2}{*}{ Score } & \multicolumn{2}{|c|}{ Quixfil (groupI) } & \multicolumn{2}{|c|}{$\begin{array}{c}\text { Ceram X } \\
\text { mono }+(\text { grou- } \\
\text { pII })\end{array}$} \\
\hline & & & (n) & $\begin{array}{c}\text { Frequen- } \\
\text { cy } \%\end{array}$ & (n) & $\begin{array}{l}\text { Fre- } \\
\text { quen- } \\
\text { cy\% }\end{array}$ \\
\hline 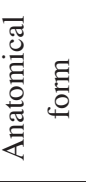 & $\begin{array}{l}\text { Baseline } \\
\text { (1 week) }\end{array}$ & $\begin{array}{l}0 \\
1 \\
2 \\
3\end{array}$ & 33 & 100 & 33 & 100 \\
\hline & 1 month & $\begin{array}{l}0 \\
1 \\
2 \\
3\end{array}$ & $\begin{array}{c}33 \\
0 \\
0 \\
0\end{array}$ & $\begin{array}{l}100 \\
0.0 \\
0.0 \\
0.0\end{array}$ & $\begin{array}{c}33 \\
0 \\
0 \\
0\end{array}$ & $\begin{array}{l}100 \\
0.0 \\
0.0 \\
0.0\end{array}$ \\
\hline & $\begin{array}{c}3 \\
\text { months }\end{array}$ & $\begin{array}{l}0 \\
1 \\
2 \\
3\end{array}$ & $\begin{array}{c}21 \\
9 \\
0 \\
0\end{array}$ & $\begin{array}{l}70 \\
30 \\
0.0 \\
0.0\end{array}$ & $\begin{array}{c}21 \\
9 \\
0 \\
0\end{array}$ & $\begin{array}{l}70 \\
30 \\
0.0 \\
0.0\end{array}$ \\
\hline & $\begin{array}{c}6 \\
\text { months }\end{array}$ & $\begin{array}{l}0 \\
1 \\
2 \\
3\end{array}$ & $\begin{array}{l}15 \\
15\end{array}$ & $\begin{array}{l}50 \\
50\end{array}$ & $\begin{array}{l}15 \\
15\end{array}$ & $\begin{array}{l}50 \\
50\end{array}$ \\
\hline & $\begin{array}{c}9 \\
\text { months }\end{array}$ & $\begin{array}{l}0 \\
1 \\
2 \\
3\end{array}$ & $\begin{array}{c}12 \\
18 \\
0 \\
0\end{array}$ & $\begin{array}{c}40 \\
60 \\
0.0 \\
0.0\end{array}$ & $\begin{array}{c}12 \\
18 \\
0 \\
0\end{array}$ & $\begin{array}{c}40 \\
60 \\
0.0 \\
0 .\end{array}$ \\
\hline & $\begin{array}{c}12 \\
\text { months }\end{array}$ & $\begin{array}{l}0 \\
1 \\
2 \\
3\end{array}$ & $\begin{array}{c}9 \\
21 \\
0 \\
0\end{array}$ & $\begin{array}{c}30 \\
70 \\
0.0 \\
0.0\end{array}$ & $\begin{array}{c}9 \\
21 \\
0 \\
0\end{array}$ & $\begin{array}{l}30 \\
70 \\
0.0 \\
0.0\end{array}$ \\
\hline & & P-valu & & $0.001 *$ & & \\
\hline
\end{tabular}




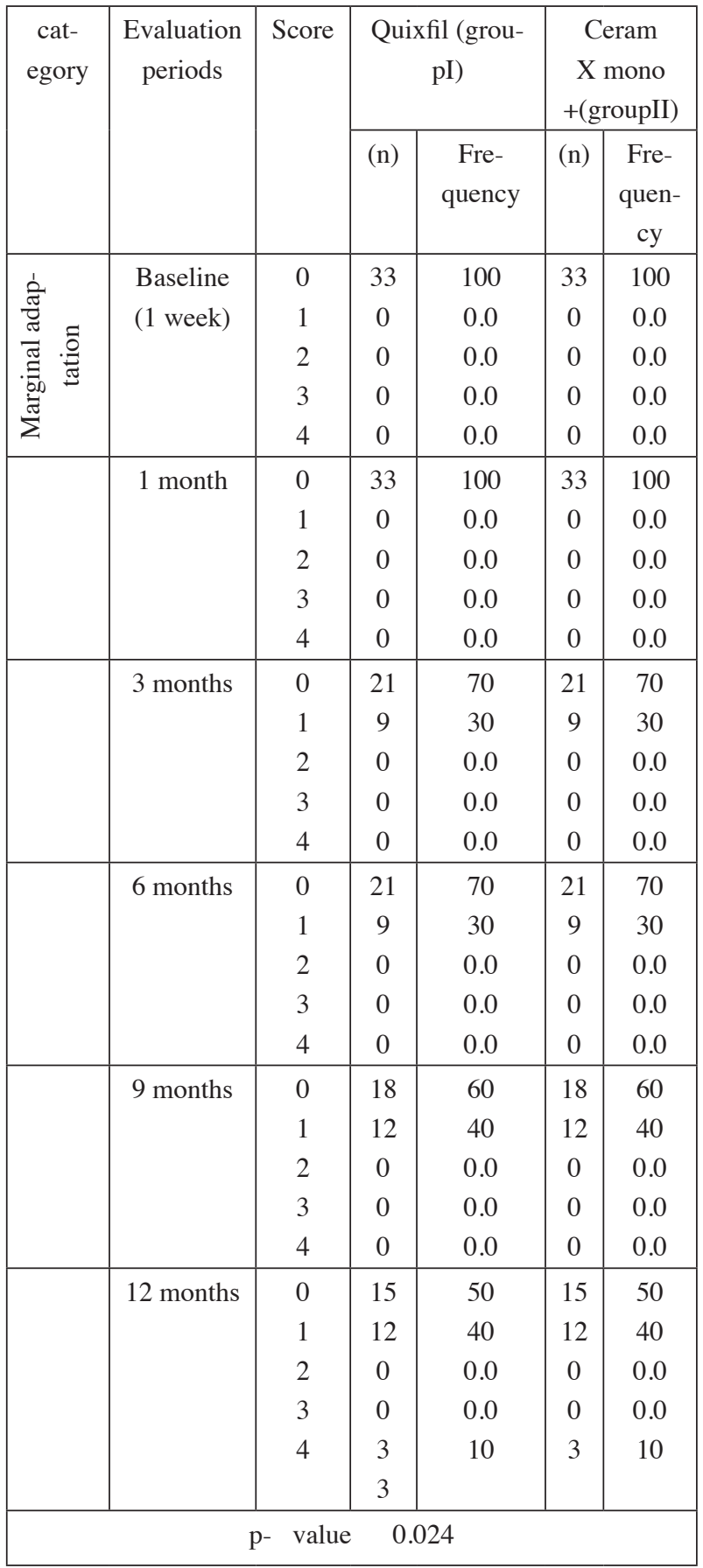

\begin{tabular}{|l|l|l|l|l|l|l|}
\hline & $(3$ & 0 & 27 & 90 & 24 & 80 \\
& months $)$ & 1 & 3 & 10 & 6 & 20 \\
& & 2 & 0 & 0.0 & 0 & 0.0 \\
& 6 months & 0 & 21 & 70 & 21 & 70 \\
& & 1 & 9 & 30 & 9 & 30 \\
& & 2 & 0 & 0.0 & 0 & 0.0 \\
& & 3 & 0 & 0.0 & 0 & 0.0 \\
\hline & 9 months & 0 & 18 & 60 & 18 & 60 \\
& & 1 & 9 & 30 & 6 & 30 \\
& & 2 & 3 & 10 & 6 & 10 \\
& & 3 & 0 & 0.0 & 0 & 0,0 \\
\hline & 2 & 0 & 18 & 60 & 12 & 40 \\
& & 1 & 6 & 20 & 12 & 40 \\
& & 2 & 6 & 20 & 6 & 20 \\
& & 3 & 0 & 0.0 & 0 & 0.0 \\
\hline
\end{tabular}

\begin{tabular}{|c|c|c|c|c|c|c|}
\hline \multirow{4}{*}{$\begin{array}{l}\text { Surface } \\
\text { roughness }\end{array}$} & \multirow{4}{*}{$\begin{array}{l}\text { Baseline } \\
\text { (1 week) }\end{array}$} & 0 & 33 & 100 & 33 & 100 \\
\hline & & 1 & 0 & 0.0 & 0 & 0.0 \\
\hline & & 2 & 0 & 0.0 & 0 & 0.0 \\
\hline & & 3 & 0 & 0.0 & 0 & 0.0 \\
\hline & \multirow[t]{4}{*}{1 month } & 0 & 33 & 100 & 33 & 100 \\
\hline & & 1 & 0 & 0.0 & 0 & 0.0 \\
\hline & & 2 & 0 & 0.0 & 0 & 0.0 \\
\hline & & 3 & 0 & 0.0 & 0 & 0.0 \\
\hline & \multirow{4}{*}{$\begin{array}{c}3 \\
\text { months }\end{array}$} & 0 & 27 & 90 & 24 & 80 \\
\hline & & 1 & 3 & 10 & 6 & 20 \\
\hline & & 2 & 0 & 0.0 & 0 & 0.0 \\
\hline & & 3 & 0 & 0.0 & 0 & 0.0 \\
\hline & \multirow{4}{*}{$\begin{array}{c}6 \\
\text { months }\end{array}$} & 0 & 24 & 80 & 21 & 70 \\
\hline & & 1 & 6 & 20 & 9 & 30 \\
\hline & & 2 & 0 & 0.0 & 0 & 0.0 \\
\hline & & 3 & 0 & 0.0 & 0 & 0.0 \\
\hline & \multirow{4}{*}{$\begin{array}{c}9 \\
\text { months }\end{array}$} & 0 & 21 & 70 & 18 & 60 \\
\hline & & 1 & 9 & 30 & 9 & 30 \\
\hline & & 2 & 0 & 0.0 & 3 & 10 \\
\hline & & 3 & 0 & 0.0 & 0 & 0.0 \\
\hline & \multirow{4}{*}{$\begin{array}{c}12 \\
\text { months }\end{array}$} & 0 & 18 & 60 & 18 & 60 \\
\hline & & 1 & 9 & 30 & 6 & 20 \\
\hline & & 2 & 3 & 10 & 6 & 20 \\
\hline & & 3 & 0 & 0.0 & 0 & 0.0 \\
\hline
\end{tabular}

\begin{tabular}{|l|l|l|l|l|l|l|}
\hline Marginal & Baseline & 0 & 33 & 100 & 33 & 100 \\
discolor- & $(1$ week $)$ & 1 & 0 & 0.0 & 0 & 0.0 \\
ation & & 2 & 0 & 0.0 & 0 & 0.0 \\
& & 3 & 0 & 0.0 & 0 & 0.0 \\
\hline \multirow{2}{*}{ (1 month } & 0 & 33 & 100 & 33 & 100 \\
& & 1 & 0 & 0.0 & 0 & 0.0 \\
& & 2 & 0 & 0.0 & 0 & 0.0 \\
& & 3 & 0 & 0.0 & 0 & 0.0 \\
\hline
\end{tabular}




\begin{tabular}{|l|l|l|l|l|l|l|}
\hline Caries & $\begin{array}{l}\text { Baseline } \\
(1 \text { week })\end{array}$ & $\begin{array}{l}0 \\
1\end{array}$ & $\begin{array}{l}33 \\
0\end{array}$ & $\begin{array}{l}100 \\
0.0\end{array}$ & $\begin{array}{l}33 \\
0\end{array}$ & $\begin{array}{l}100 \\
0.0\end{array}$ \\
\hline & $(1$ month $)$ & 0 & 33 & 100 & 33 & 100 \\
& 1 & 0 & 0.0 & 0 & 0.0 \\
\hline & $(3$ months $)$ & 0 & 30 & 100 & 27 & 90 \\
& & 1 & 0 & 0.0 & 3 & 10 \\
\hline & (6 months $)$ & 0 & 30 & 100 & 27 & 90 \\
& & 1 & 0 & 0.0 & 3 & 10 \\
\hline & (9 months $)$ & 0 & 30 & 100 & 27 & 90 \\
& 12 months & 0 & 27 & 90 & 24 & 80 \\
& & 1 & 3 & 10 & 6 & 20 \\
\hline
\end{tabular}

\section{DISCUSSION}

In this study The modified USPHS criteria wellknown method realistic for evaluation of restoration in clinical studies ${ }^{(4)}$. It is the commonly - used system for assessing the important characters of dental restorations ${ }^{(7)}$, such as marginal adaptation, marginal discoloration, surface roughness and secondary caries. Satisfactory to confirm that restorations made in the same conditions, one experienced dentist placed all the restorative martials in all cavities in this trial, this is agrees with ${ }^{(8)}$. (Bayraktar et al., (2016) And disagrees with ${ }^{(9)}$ (Faye et al., (2015) who were performed restorations by more than one technician which decreased the exactness of technique and these variables were in need of operator than on the material tested. Flowable resin composite used in this trial as intermediate layer between packable composite and tooth structure, this for decreasing filler and enhancing the flow capacity which lead to decreasing the stresses and increasing the sealing ability these findings agreed with ${ }^{(10)}$ (Kucukyilmaz et al., (2016). Layering placement techniques is required to gain adequate light penetration and to polymerization shrinkage ${ }^{(11)}$. Standard technique $2 \mathrm{~mm}$ increments for composite placement, each of them exposed to separate light curing. This technique takes more time and may lead to increase contamination of restoration which may include voids between layers also, incremental filling technique increase stress concentration due to oblique layering that increase microleakage, debonding, secondary caries and post-operative pain ${ }^{(12)}$. Additional increments increased the cuspal deformation (13). Furthermore, it was stated that multi-step bonding systems caused gaps in the interface between resin and dentin, these gaps cause microleakage and postoperative sensitivity ${ }^{(14)}$. Bulk filling resin composite is a simplified fewer steps technique in placement of composite resin which more preferred in children, it allows curing in $4 \mathrm{~mm}$ layers due to translucency of material and integration of a photoactive group of methacrylate resin lets resin for curing at $4 \mathrm{~mm}^{(15)}$. There is no statistically significant differences were present between the packable bulk fill and Nano hybrid composite for marginal adaptation, surface roughness and caries detection parameters at all periods of evaluation, so the null hypothesis was established in these parameters. It may be due to translucency of bulk fill composite for light curing more than traditional composite resin furthermore, it has large filler size and therefore dropping the specific surface between fillers and organic matrix that decrease the light scattering ${ }^{(19)}$. The clinical results of packable Bulk filling technique 4-mm is highly -satisfactory when it compared to the conventional 2-mm incremental technique ${ }^{(16,17)}$. These outcomes were detected in current study that compare performance of bulk fill composite with nano hybrid composite in class I and II restoration. ${ }^{(18)}$

\section{CONCLUSION}

Packable bulk fill composite resin is more simply and easy as a tooth colored restorative material for children. One placement layer for packable bulk fill shows acceptable clinical results when compared with nano hybrid composite at different evaluation periods (1week, 1 month, 3 month, 6 month, 9 month and 12 month) 


\section{RECOMMENDATION}

Future studies should evaluate restorations over a longer timescale to determine the long-term clinical performance of packable bulk-fill resin composite materials in children.

\section{ACKNOWLEDGMENTS}

Thanks to all children and their families who participate in this study

\section{REFERENCES}

1- Sunnegardh- Gronberg K, van Dijken J Funegard U et al. Selection of dental materials and longevity of replaced restorations in public dental health clinic in north Sweden . J Dent. 2009; 37:673-8.

2- Demarco FF, Gorrea MB, Cenci MS et al. Longitivitiy of posterior composite restorations :not only a matter of materials. Dent Mater.J. 2012;28:87-101 .

3- Poskas L, Placido E, Cardoso P. Influence of adhesive system and placement technique on microleakage of resin based composite restorations. J Adhes Dent. 2004 ; 6:227-32.

4 - Campodonico C, Tantbirojn D, Olin P. Cuspal deflection and depth of cure in resin- based composite restorations filled by using bulk, incremental and transtooth -illumination techniques. J Am Dent Assoc. 2011;142:1176-82.

5- Park J Chang J F errancane J . How should composite be layered to reduce shrinkage stress: inceremental or bulk filling . Dent Mater J. 2008;24:1501-5.

6- Benetti A, Havndrup C, Honore D et al. Bulk fill resin composites : polymerization contraction, depth of cure , and gap formation . Oper dent. 2015;40:295-303.

7- de Andrade AK, Duarte RM, Medeiros e Silva FD . 30 month randomized clinical trial to evaluate the clinical performance of a nanofill and nanohybrid composite. J Dent. 2011;39:8-15.

8- Bayraktar Y, Ercan E, Hamidi M, Colak H. One -year clinical evaluation of different types of bulk -fill composites. J Investigative and clinical Dentistry. 2016;0:1-9.
9 - Faye B, Sarr M, Bane K. One year clinical evaluation of the bonding effectiveness of a one - step , self -etch adhesive in noncarious cervical lesion therapy. Int J Dent. 2015;2015:984065.

10- Kucukyilmaz E, Yasa B, Ackcay M, Savas S, Kavrik F. Effects of pulp capping materials on fracture resistance of class II composite restorations. European Journal of Dentistry . 2016;9:218- 23.

11- Feilzer AJ, de Gee AJ, Davidson CL. Setting stress in composite resin in relation to configuration of the resetoration. J Dent Res. 1987;66:1636-9.

12- Abbas G, Fleming GJ, Harrington E, Shortall AC, Burke FJ. Cuspal movement and microleakage in premolar teeth restored with a packablen composite cured in bulk or incerements. J Dent .2003;31:437-44.

13- Versluis A, Douglas WH, Cross M, Sakaguchi RL. Does an inceremental filling technique reduce polymerization shrinkage stresses. J Dent Res. 1996;75:871-8.

14- Opdam NJ, Feilzer AJ, Roeters JJ, Smale I. Class I occlusal composite resin restorations in vivo post- operative sensitivity, wall adaptations, and microleakage . Am J Dent. 1998;11:229-34.

15- Jin X, Bertrand S, Hammesfahr PD. New radically polymerizable resin with remarkably low curing stress. J Dent Res. 2009; 88(spec issue A): 1651.

16- Bayraktar Y,Ercan E, Hamidi M, Colak H. One -year clinical evaluation of different types of bulk -fill composites . J. Investigative and clinical Dentistry. 2016;0:1-9.

17 - Van Dijiken JW, Pallesen U. A randamized controlled three years evaluation of bulk filled posterior resin composites restorations based on stress decreasing stresses technology. Dent Mater. J. 2014; 30:e245-51.

18 - Van Dijken JW, Pallesen U. A randomized 3-year clinical evaluation of class I and II posterior resin restorations placed with a bulk -fill resin composite and one- step selfetching adhesive. J Adhes Dent. 2015; 17:81-8.

19 - Finan L, Palin W.M.,Moskwa N.,Mcginley E.L.,Fleming G.L. The influence of irradiation potential on the degree of conversion and mechanical properties of two bulk fill flowable RBC materials .Dent Mater. J. 2013;9:906- 12. 


\section{Patient informed consent}

Name of patient:

Age:

Sex:

Name of parent: Phone number:

Name of research: - One Year -Randomized Clinical Trial to Evaluate Packable Bulk fill and Nano Resin Composite in Children and Adolescents

1- Type of research: - clinical research.

2- Subject of research: - children $<18$ years.

3- Request is being made to give up informed consent.

4- The research is for the good of society.

5- Study design: - randomization

6- Facilities of research are available

7- List of risks of the study:

- Pain -Fracture restoration - recurrent caries

\section{8- Lists of potential benefits to the subjects:}

- Removal of caries - restoration of permanent first molar .

9- Privacy and confidentiality of subjects are assured.

10- The subject of the research could quit at any time without penalty or loss of any benefits to which they would otherwise be entitled.

Signature of principal investigators Parent signature 